\title{
Макєठоovıкá
}

Tóp. 10, Ap. 1 (1970)

\section{L' hospice Grec pour les indigents de Pest}

\section{MAKGAONIKA}

IYTГPAMMA IIEPIOAIKON

THE ETAIPEIAZ MAKEAONIKSN EHOY

EIIMEAEIA,

A. KANATEOYAH - $\Sigma$. ПAIIASOПOYAOY - $\bullet$. ПETEA

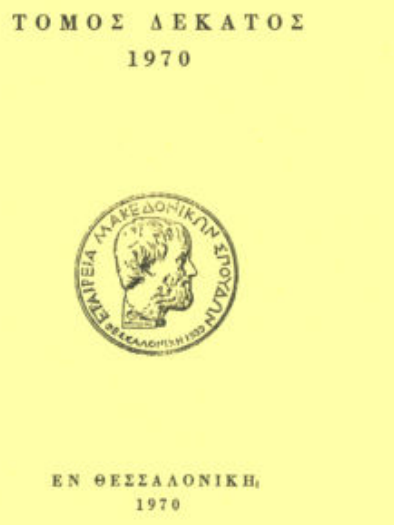

Ödon Fuves

doi: $10.12681 /$ makedonika. 777

\section{Copyright $@$ 2014, Ödon Fuves}

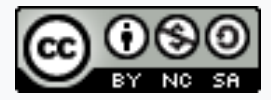

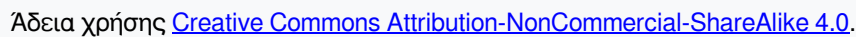

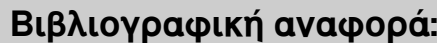

Fuves, Ödon. (2015). L' hospice Grec pour les indigents de Pest. Макєঠovıкá, 10(1), 289-291. https://doi.org/10.12681/makedonika.777 


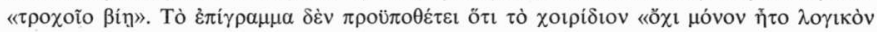

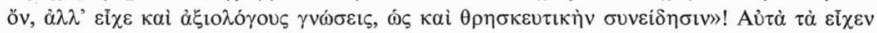

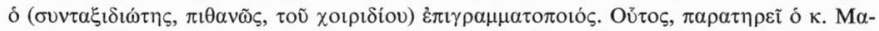

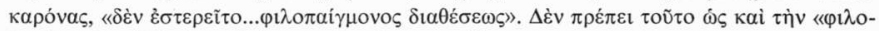

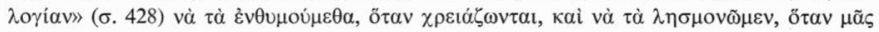

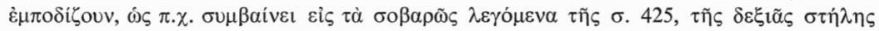

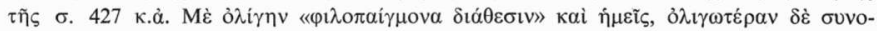

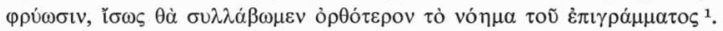

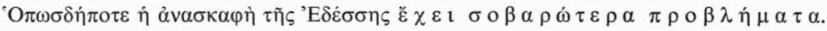

\section{THE EPIGRAM FROM EDESSA AGAIN}

It has been stated earlier ${ }^{2}$ that the Edessa tombstone is that of a young pig killed while being brought to Emathia. The pig is named, described and pictured-once while travelling and again while being run over. The epigram tells us a story which exactly coincides with the illustration. The evidence for my interpretation is based on the obvious and it takes no flight of fancy to be substantiated.

Imaginative interpretations have been given by others ${ }^{3}$, based on a possible meaning of one word or another. The fact that the one word can be interpreted in many ways proves that it is unsafe to take recourse solely in imagination (see p. 300).

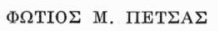

\section{L'HOSPICE GREC POUR LES INDIGENTS DE PEST}

Au XVIIIe siècle Pest était le centre des Grecs de culture grecque, arrivés en grand nombre en Hongrie pour faire des affaires. Ici, ces riches marchands se firent bâtir une église à part sur le bord du Danube et pour leurs enfants instituèrent une école à part. Mais ils n'oublièrent pas leurs vieillards malades. De fait, dans leurs testaments plusieurs négociants ont légué des sommes plus ou moins grandes pour ce but noble 4 .

Mais pour s'occuper davantage et prendre soin des veillards d'une façon constante et institutionelle, on décida déjà en 1787 la création d'un hospice pour les pauvres. Dans ce but on organisa une collecte. Une des plus grandes sommes, 10.000 (dix-mille) florins fut léguée encore cette année par testament par Nicolas Paziazi pour la fondation de l'hôpital. Rien n'ayant encore été résolu jusqu'en 1793 au sujet de ce legs, le Conseil de Lieutenance

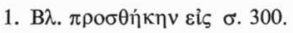

2. AAA II (1969), p. 190.

3. AAA II (1969), pp. 422-429.

4. A n a s t a s T h e o dos i a légué en 1775 de l'argent pour l'hôpital communal (Archives de la capitale - Inventaria et Testamenta s. a. 1187).

Un document datant de 1790, gardé dans les archives de la communauté ecclésiastique hongroise orthodoxe de Budapest consigne sur une liste les instruments et objets d'équipement manquant à l'hôpital. Il est certain que les Grecs ont aussi contribués par leurs dons à les procurer à l'établissement. 
(gouvernement local) demanda au Conseil municipal ce qu'il faut faire avec cette somme ${ }^{1}$.

Le magistrat déclara qu'il a l'intention de consacrer cet argent à la fondation de l'hospice municipal où seraient placés les viellards et les indigents grecs. Sur cela le Conseil de lieutenance demanda l'avis des Grecs qui, bien entendu, se prononcèrent aussi pour un hôpital indépendant ${ }^{2}$.

En 1794, les Grecs aménagèrent au rez-de-chaussée du bâtiment de l'école de la rue Galamb-acheté 5 années auparavant des Piaristes-l'hôpital comprenant 3 chambres. Dans l'une, on dressa 5 lits pour les malades alités, une autre pièce fut réservée aux convalescents et la troisième à l'infirmier.

Dans son rapport, rédigé comme suite à l'inspection faite sur place le 13 mars, le médecin Joseph Csehszombathy certifia que les chambres sont convenables et que la communauté ecclésiastique a assumé l'équipement entier de l'hôpital. Selon l'avis du médicin de l'Etat civil, les frais d'entretien d'un lit seront environ 125 florins par $a^{3}$.

En dehors des malades alités, l'hôpital a soigné aussi des viellards et personnes agées malades. C'est pourquoi le titre complet de cet établissement était en allemand «Spital und Armeninstitut» en latin «Hospitialis domus». Dans les textes grecs à côté de la forme grecque «szpitali» de ce mot latin (hospitialis domus) figurent les dénominations: xenodochion, orfanotrofion, ptochotrofion et nosokomion».

A la tête de l'hôpital se trouvait un administrateur (gérant) élu pour deux ans par la communauté ecclésiastique. C'est lui qui était chargé de la comptabilité de l'hôpital. Les actes et écrits principaux relatifs à l'hôpital étaient placés dans les Archives de l'institution. Si quelque tension survenait dans les rapports entre les Grecs, il arrivait que même deux administrateurs fonctionnaient en même temps. L'hôpital disposait d'un médecin permanent qui tenait registre des recettes prescrites aux malades dans un carnet d'ordonnances ${ }^{4}$. Les malades étaient soignés et surveillés par un infirmier, qui était lui-même aidé par un domestique.

La tâche de l'administration de l'hôpital était l'aide regulière et systématique des Grecs appauvris, qui en avaient besoin de même que l'assistance éventuelle aux voyageurs et réfugiés (Versorgungsinstitut). C'est lui qui présentait à la direction de la communauté ecclésiastique les demandes de secours et c'est aussi lui qui, après la décision remettait l'allocation de secours à l'intéressé.

En 1821 Schams écrit de «l'hôpital des Grecs non unis» qu'il comprend 2 pièces: l'une pour les hommes, l'autre pour les femmes ${ }^{5}$.

En 1828-1829 l'hôpital comptait 7 lits et l'entretien en était confié à une femme et à sa fille.

1. A r chives $\mathrm{Nat}$ io n a les - Archives du Conseil de la lieutenance Départ Relig. Graec. n. a. 1793 fons 49 Ordonnance 1793, III, 7 -1, 7078 du Conseil de la lieutenance.

2. Lors de la visite à Budapest de Townson Robert en 1793, il n'y avait que deux hôpitaux dans la capitale: l'hôpital de l'Université avec 12-15 places et l'hôpital de la Ville avec 8 lits. Tous les deux étaient dans un mauvais état, négligés.

3. Archives de 1 a Ville (Capitale), Intimata a. m. 5539.

4. Les livres de recettes (médicaments) des années 1831-44, 1861-74 et 1789-90 se trouvent dans les archives de la communauté ecclésiastique hongroise orthodoxe de Budapest.

5. F. S c h a ms, Vollständige Beschreibung der Königlichen Freystadt Pest in Ungarn, Pest 1821, pp. 325-326. 
En 1836 nous trouvâmes le reglement concernant les malades et le personnel écrit en grec.

En 1838 dans l'une des chambres de l'établissement il y avait de la place pour 6 hommes, dans l'autre pour 6 femmes'.

En 1840 un contrôle constata que la maison pour indigents était bien aménagée mais très sale. La chambre pour hommes était spacieuse, mais celle des femmes exiguë, comme une prison. C'est dans cette année, qu'après le Dr Birly, le Dr Emmanuel Ferczy, d'origine grecque assuma la direction de l'hôpital.

En 1841 l'hôpital fut transféré dans l'aile du Sud du corps de bâtiment construit alors au bord du Danube faisant partie de la «Cour Grecque» (sic).

En 1854 l'hospice pour indigents disposait de 8 lits mais on y assistait en outre 40 pauvres.

Faute de données et documents, nous ne savons pas la date de la cessation de cet établissement. Mais comme en 1908 nous avons trouvé une note (facture) dressée pour l'hospice des pauvres, nous pouvons en conclure que cette noble institution a encore existé au commencement de ce siècle.

Budapest

ODÖN FUVES

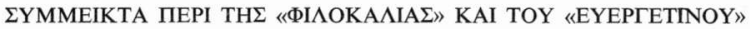

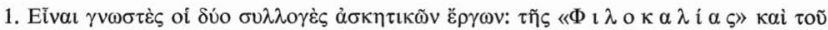

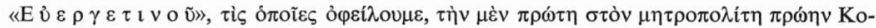

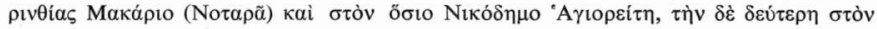

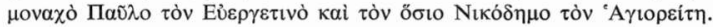

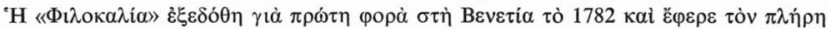

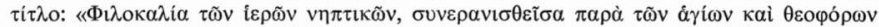

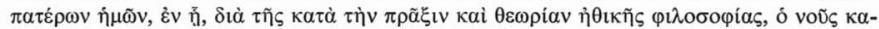

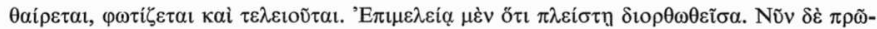

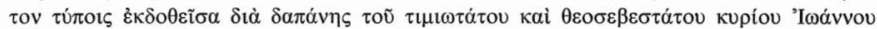

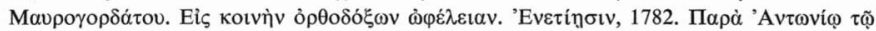

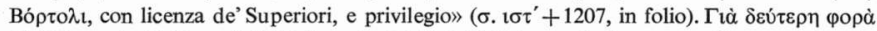

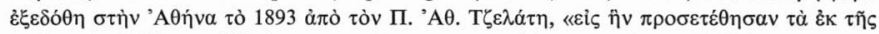

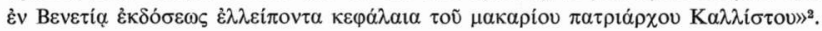

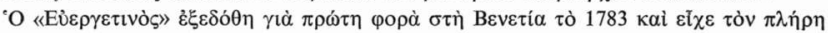

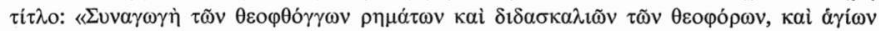

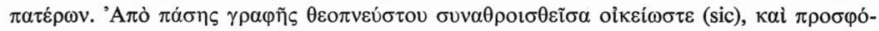

1. Englenderné Brüll Klára, Orvosok és Kórházak Pest-Budán Bp. é,n. 118-119 (Mme Englender-Brüll Klára: Médecins et hôpitaux de Pest-Buda).

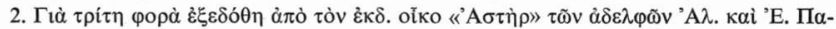

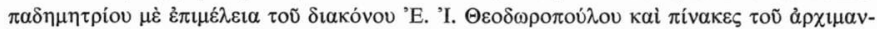

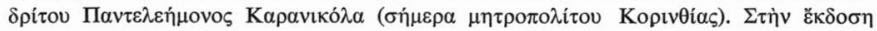
$\alpha$

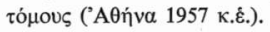

